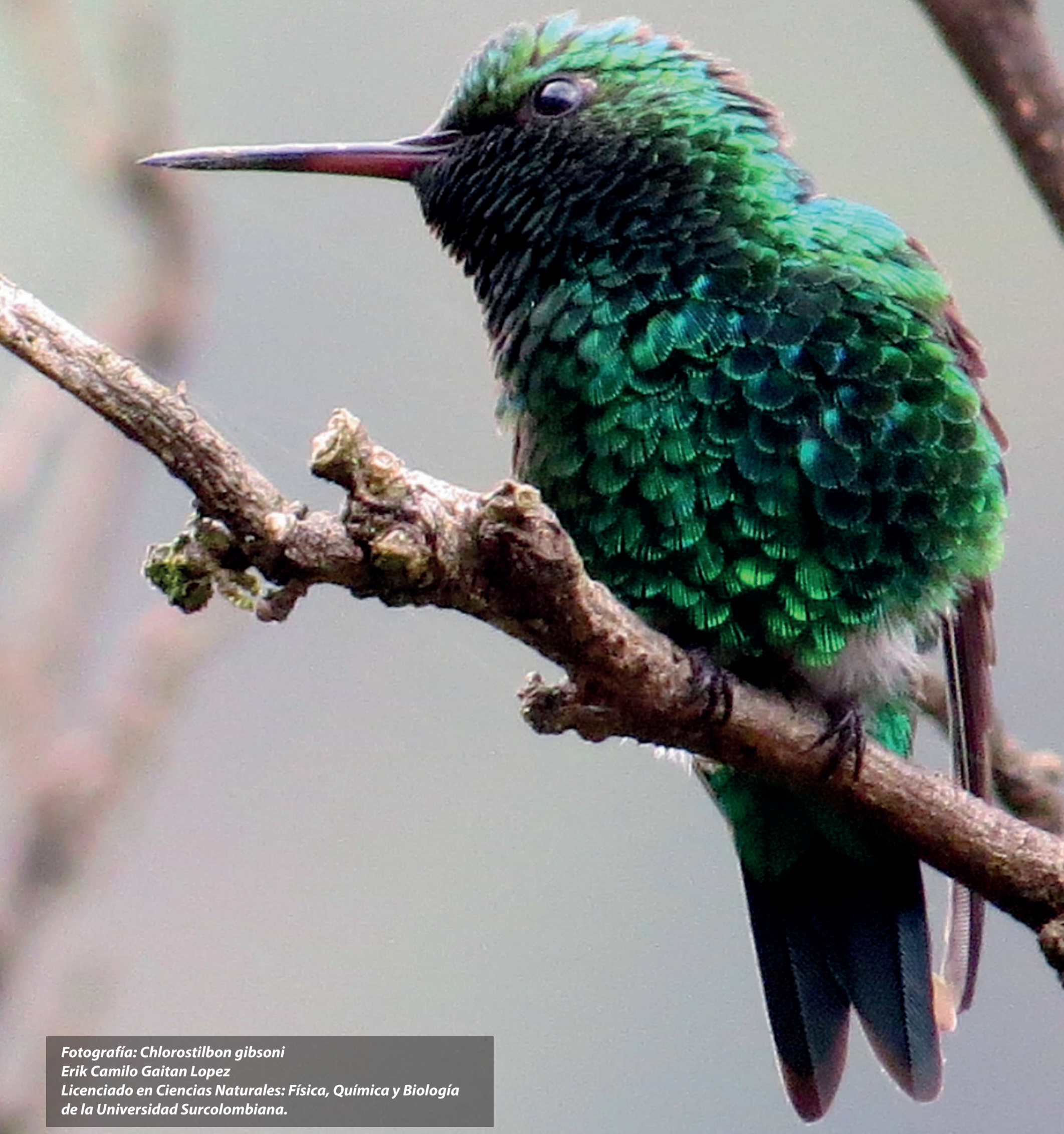




\title{
MATICES, PLIEGUES E INCITACIONES ENTRE LA BIOLOGÍA Y LA PEDAGOGÍA EN LA LICENCIATURA EN BIOLOGÍA DE LA UNIVERSIDAD PEDAGÓGICA NACIONAL
}

\section{SHADES, FOLDS AND INCITEMENTS BETWEEN BIOLOGY AND PEDAGOGY \\ The degree in biology from the National Pedagogical University}

\author{
Sandra Liliana Cano Aparicio ${ }^{1}$ \\ Carold Andrea Hernández Gómez ${ }^{2}$
}

Fecha de recepción: 28 de marzo de 2014

Fecha de aprobación: 22 de abril de 2015

\section{Resumen}

Este documento refleja un ejercicio investigativo desarrollado con elementos de la caja de herramientas del Grupo Historia de la Práctica Pedagógica y de la perspectiva foucaultiana. En este caso, a partir de un rastreo de fuentes y registros se problematizó la relación entre biología y pedagogía en la Licenciatura en Biología de la Universidad Pedagógica Nacional. Dicha relación se configuró con fuerzas, choques, matices, resistencias, que permitieron visibilizar tres pliegues y momentos distintos.

Igualmente, más allá de la licenciatura que nos compete, este escrito problematiza las tensiones, pliegues, movilizaciones y luchas que se producen cuando entran las disciplinas o ciencias en conexión con la pedagogía. Ade- más, se visibiliza unas prácticas singulares con relaciones de saber, poder, subjetividades diferentes, que denotan una práctica creadora, política y estética de la Licenciatura en Biología.

\section{Palabras clave:}

Pedagogía; biología; saberes; poder; maestros/as

1 Licenciada en psicología y pedagogía de la Universidad Pedagógica Nacional. (Colombia) Línea de investigación formación pedagógica, formación de maestros. Correo electrónico: Sandralilianacano@yahoo.com

2 Licenciada en psicología y pedagogía de la Universidad Pedagógica Nacional (Colombia) Línea de investigación formación pedagógica, formación de maestros. Correo electrónico: andidi22@hotmail.com 


\section{Abstract}

This document reflects an investigative exercise developed from elements of the toolbox of the History Group of the Pedagogical Practice and Foucauldian perspective, in this case from a source tracking and records the relationship between biology and pedagogy problematized Degree in biology from the National Pedagogical University. This relationship emerged with force, shock, nuances, resistors which gave some insight trifold and different times.

Also, this paper problematizes from a Bachelor tensions, folds, demonstrations and struggles that occur when entering or science disciplines in connection with pedagogy. Furthermore, some singular relations practices of knowledge, power, subjectivity different denoting a creative practice, politics and aesthetics of Bachelor's degree in biology

\section{Keywords:}

Pedagogy; biology; knowledge; power; teachers/as

La mirada que orientó este trabajo de investigación se articuló a partir de la caja de herramientas metodológicas que utiliza el Grupo Historia de la Práctica Pedagógica en Colombia, que en gran medida surge de la lectura juiciosa y de la extracción de nociones y elementos del trabajo foucaultiano aplicables a la investigación. A través de este enfoque se buscó mirar la historia no desde una linealidad cargada de discursos hegemónicos o de relaciones causa-efecto; esto con el fin de entrar a problematizar los juegos de verdad y las relaciones que se mantienen, transforman, permanecen o han sido establecidas como resultado de un conjunto de fuerzas que son anónimas, pero identificables.

Cabe destacar que en la construcción de este trabajo se utilizaron dos tipos de fuentes: las primarias y las secundarias $^{3}$. Esto supuso una localización, recolección y rastreo de diferentes tipos de documentos y registros. En el acercamiento a ellos, se lograron desarticular temáticas y categorías para así, visibilizar las regularidades, las discontinuidades, la dispersión y los puntos singulares de

3 En este tipo de metodología los dos tipos de fuentes son importantes pero diferentes, por un lado las Fuentes primarias atañen a los documentos que fueron leídos para identificar las temáticas, enunciados y la caracterización con la cual se estableció el diagrama de tendencias que actualmente se dan en la enseñanza de la biología, la formación de maestros/ as y el tipo de relación de este campo disciplinar con la pedagogía. Mientras que las fuentes secundarias: Conciernen a los textos, publicaciones, lecturas abordadas que complementan o tensionan lo hallado en las fuentes primarias. encuentro donde convergen los momentos históricos y donde se hace visible la relación buscada, a partir de unos intereses y unas necesidades específicas.

Igualmente, las reflexiones que se plasman en este documento se enmarcan en un momento álgido de polémicas y demandas que recaen sobre el maestro y las instituciones formadoras de educadores. El bajo estatus de la profesión docente, las concepciones reduccionistas que muestran el papel del maestro/a como un repetidor de discursos y las políticas educativas que asumen que cualquier profesional con un curso de pedagogía puede enseñar o que para ser un buen profesor sólo se necesita tener un adecuado dominio del componente disciplinar, se situaron como condiciones para empezar a configurar y problematizar, a partir de una licenciatura (en este caso en biología) las resistencias, pliegues y luchas que ocurren cuando entra en conexión una disciplina con la pedagogía.

De este modo, los pliegues que configuraron este documento permitieron hacer visible un encuentro de la pedagogía con la biología que se ubica de forma distinta en tres momentos y que a través de líneas de fuerza constituye modos de ser sujeto, escenarios y saberes particulares.

\section{Primer pliegue. Educación para la salud: la pedagogía como estrategia}

Es significativo resaltar que la noción de salud ha sido transversal desde la creación de la Licenciatura hasta la actualidad; sin embargo, se han dado modos de circulación y prácticas distintas en cada época. Con la revisión de los acontecimientos que la configuraron y la han constituido, se develaron los discursos que influyeron en la forma de ser de la Licenciatura en Biología, lo que permitió un acercamiento a las relaciones, ya sea de imposición, resistencia o incitación entre los sujetos, las instituciones y los saberes.

En este primer periodo se le da un lugar diferente a la educación del niño, pues sobre él recae la posibilidad de construir un hombre distinto, de modo que al educarlo, al enseñarle prácticas higiénicas, controlarlo y disciplinarlo se alcanzaría una población fuerte y saludable en aras de un desarrollo socioeconómico para la nación. Estos hechos llevarían a la Licenciatura a emprender un trabajo de preparación de los futuros educadores para que escudriñaran con el fin de alcanzar esas metas trazadas. Así, el educador se convirtió en el guía de la salud. 


\section{El proyecto de una sociedad fuerte y sana, a través de la higiene y la salud}

En Colombia, fundamentalmente a partir de 1918, surge la necesidad de la higiene en toda la población, según Carlos Noguera (2003). A partir de su archivo de documentos, el autor data en esa fecha la llegada de la denominada "peste" o gripe al país, la cual ocasionó una gran cantidad de víctimas e incitó transformaciones que iban desde la construcción y ampliación de cementerios, hospitales de emergencia y un manejo sanitario para la ciudad, hasta la reflexión sobre las estrategias y mecanismos necesarios para higienizar a la población, principalmente al obrero.

Las elites, lejos de una verdadera preocupación por lo que sucediera al "pueblo apestado" (Noguera, 2003), encontraban que sus asentamientos eran un foco de microorganismos en los cuales pululaban las enfermedades que se propagaban no sólo entre ellos sino que fácilmente alcanzaban a la alta sociedad, en este sentido, tenían que encontrar la manera de contrarrestar estos hechos abrumadores; aparecía la educación como una estrategia primordial.

En correspondencia con lo anterior, la Licenciatura en Biología entró en conexión con la sociedad y sus requerimientos, desarrollando cátedras en las que el eje motivacional se dirigió a reflexionar y aportar transformaciones que mejoraran a la sociedad colombiana. Un claro ejemplo lo constituyó el programa Educación para la Salud, cuyos objetivos estaban orientados a presentar los beneficios de la salud en el país: "Identificar y evaluar las condiciones de salud de la población colombiana y diseñar un plan de participación y apoyo a los programas existentes para el mejoramiento de la salud" (Departamento de Biología, 1973, p. 2).

Esta perspectiva mostró que para la Licenciatura el enfoque era brindar mejoras a la sociedad. No obstante, otros intereses también movilizaron dichos propósitos: el Estado colombiano generó aparatos para la administración de la biopolítica, fundamentalmente a partir de 1855. Dichos aparatos, la escuela entre ellos, buscaban conocer y gobernar las manifestaciones de la vida interviniendo en los sujetos a través de higiene, estados de salud, educación, economía doméstica entre otros, tal como lo resalta Pedraza (2004, p. 186).

Uno de los referentes principales que usaba la Licenciatura en biología fue el libro "Puericultura e higiene escolar", El texto estaba constituido por dos secciones: la primera, dedicada a la puericultura, describía minuciosamente horarios, nutrición, enfermedades y cuidados para los niños. La segunda parte, orientada a la higiene escolar, trataba sobre limpieza de las habitaciones, primeros auxilios, enfermedades comunes que deben ser detectadas por los maestros, las características de la escuela, del buen maestro e, incluso, especificaba las condiciones arquitectónicas y del mobiliario que se debía utilizar para evitar enfermedades del cuerpo humano (Mendoza, 1965).

A nivel general, los catálogos bibliográficos que usaba la Licenciatura hacían alusión a escenarios como sociedad, familia, matrimonio, y provenían de la Asociación Colombiana de Facultades de Medicina, La Cruz Roja Colombiana, la Organización Mundial para la Salud; pues la opinión y los textos que producían dichas instituciones eran relevantes en los aprendizajes y contenidos enseñados a los maestros.

\section{Maestro de biología vigía y guía para la salud}

En este primer momento, el licenciado en biología se nombraba como el educador para la salud, con unas connotaciones y requerimientos particulares. Clara evidencia de ello son los objetivos de los programas de educación para la salud de 1973, en los que el educador debía identificar el papel que le correspondía en la solución de los problemas de salud del país y elaborar planes de acción realizables para lograr este fin. Al prevalecer la preocupación por la vida, el rol del educador tenía un marcado compromiso con la salud y con el propósito de orientar a sus educandos en el cambio de actitudes y hábitos en relación con esta.

Conjuntamente, en el programa de posgrado en educación para la salud, se desarrolla exhaustivamente el área de salud mental, dictada en su totalidad por una enfermera y dos médicos psiquiatras, en dicho programa se considera al pedagogo como:

Elemento responsable de la formación y capacitación de los elementos jóvenes del grupo, tiene un papel a desempeñar muy importante dentro del contexto de la salud y de la salud mental específicamente; en sus manos la comunidad ha depositado la prolongación, conservación, transmisión de conocimientos, desarrollo de los valores individuales, de la familia, comunidad, nacionalidad [...]. (Olivares, Luna, y Delgado, s. f., p. 1)

Lo anterior muestra una fuerte inclinación de formar a los licenciados/as en biología a partir de principios de medicina y enfermería, evidenciado en los textos que circularon en seminarios de la época: microbiología para enfermeras, el control de las enfermedades, medicina para todos, compendios de psiquiatría y psicoanálisis, higiene mental, psiquiatría clínica, manuales de terapias, de psicopatología, de enfermería y de primeros auxilios, guías para la




salud y enseñanza de la higiene, entre otros; temas que le daban este tinte al maestro de biología.

\section{Pedagogía como estrategia de promoción y prevención para la salud}

En primer lugar, en este pliegue (Figura 1) el objeto de la biología más que el estudio de lo vivo es la preocupación por la vida. Así, desde la Licenciatura en Biología, al hablar de anatomía, fisiología humana, biología vegetal y parasitología, se hizo imprescindible dar un trasfondo a la salud pública, a la educación sanitaria, a la industria y, en general, a las condiciones de la población colombiana; noción alrededor de la cual se fueron adhiriendo otra serie de saberes que le dieron un modo de ser distinto a esta formación de futuros licenciados.

En segundo lugar, la educación, tal como la describen Martínez, Noguera y Castro (1994), logra traspasar las fronteras de la universidad y para estos inicios de la Licenciatura en Biología se observa claramente que estaba impregnada por el deseo de progreso, desarrollo socioeconómico y además consolidar en el país una población saludable con una conexión fuerte entre la biología y saberes como la medicina.

El cuerpo aparece en esta época como el lugar sobre el cual recaen diversos ejercicios de control. Se piensa que los cuerpos débiles, desnutridos y enfermos son focos de enfermedad y, por ende, son degenerados. Al respecto, se requiere de una intervención a través de la cual se higienicen, no solo hablando de limpieza, sino de nutrición, posturas acordes, mobiliarios, prácticas sanas y arquitectura pertinentes para un nuevo mundo industrializado que se estaba gestando en el país. En la Licenciatura en Biología estuvieron latentes todos estos rasgos, dado que desde allí se posibilitaría alcanzar una multiplicación de dichos conocimientos hacia la población.

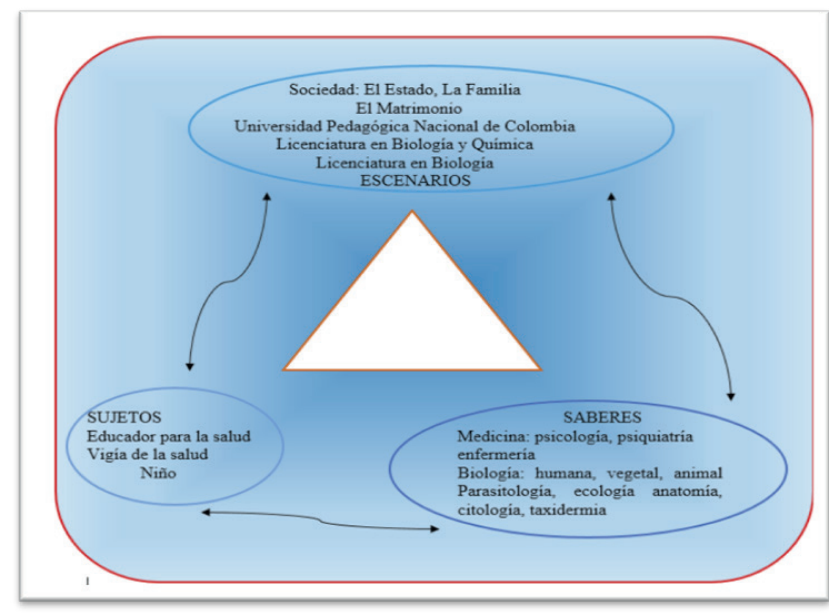

Figura 1. Primer pliegue educación para la salud: la pedagogía como estrategia.

\section{Segundo pliegue. Nuevas cartografías y pedagogías}

El segundo momento y pliegue que emerge de la relación entre la biología y la pedagogía está atravesado por dos procesos claves: el movimiento y la expedición pedagógica. Estos sucesos movilizaron reflexiones que permitían explorar la vida cotidiana de la escuela y las relaciones de esta con el entorno sociocultural, el territorio, la práctica política y las experiencias de los maestros/as en todo el país. Además, estas expresiones de resistencia resignificaron el estatuto del maestro y fueron un llamado a construir una nueva pedagogía que trascendiera las prescripciones curriculares que habían sido impuestas por modelos educativos ligados al desarrollo.

Lo anterior fue expresión de una transformación pedagógica y social que permeó escenarios, prácticas educativas, proyectos políticos y subjetividades. Uno de los escenarios que emerge con fuerza en la desarticulación de los documentos es el Departamento de Biología de la Universidad Pedagógica, que, a mediados de 1984, en cabeza de profesores como Rosalba Pulido de Castellanos, Guillermo Chona, Fanny Forero, Margie Jessup Cáceres, Norma Constanza Castaño, entre otros, empezó a interpelar cómo armonizar lo pedagógico y didáctico con lo disciplinar y aclarar las relaciones existentes entre las estrategias científicas de la disciplina y la enseñanza de la misma. Estos análisis posibilitaron un viraje del proyecto curricular de la Licenciatura hacia 1999, fortaleciendo las áreas de formación pedagógica y didáctica y la integración de esta con la formación específica y otros saberes, lo que conformó un sistema de interacciones que provocaron la aparición de otras formas de nombrar al licenciado de biología. Se desplazó la connotación de Educador para la salud y se situó como un sujeto intelectual e investigador que produce saberes anudados a la categoría de territorio, experiencia y corporalidad, con una preocupación más por lo vivo y los elementos que marcan la vida de los organismos.

Se ubicó a la pedagogía como una nueva escritura, una coordenada de análisis y una fuerza de incitación que problematiza y hace otras lecturas del saber biológico, de la enseñanza de la biología y de lo que implica ser licenciado en este campo disciplinar. Además, se abrió la posibilidad de deconstruir la relación jerárquica que asume que la ciencia y la disciplina son las únicas que aportan y consolidan saberes en las universidades y en las escuelas, que los contenidos que se enseñan en estos espacios vienen de afuera y que estos lugares solo actúan como reproductores de conocimiento; para mostrar desde la práctica y la experiencia que hay otras formas de asumir la biología y que aquello que se produce en espacios como la escuela 
y la universidad tiene propósitos e intereses distintos a los que se mueven en las comunidades científicas.

\section{El maestro artesano de la pedagogía: constructor y arquitecto de saberes}

Las implicaciones políticas, culturales, sociales y económicas que tiene la biología principalmente relacionadas con la ecología, la salud, la conservación, la biodiversidad y el mejoramiento de la vivencia humana, posibilitaron hablar de un licenciado en biología con una connotación de maestro; un maestro revestido de ciertas características que hacen que su perfil profesional tome particularidades específicas de acuerdo a los retos y responsabilidades que asume la enseñanza de la biología.

Desde la construcción del nuevo perfil profesional, que se inició con una serie de reflexiones a partir de 1982 y que se consolidó hacia marzo de 1999 con la presentación del proyecto curricular que establecía como ejes trasversales la formación en el desarrollo integral humano y la investigación, emerge la noción de un maestro no como un administrador del currículo que actúa como ejecutor de planes y diseños instruccionales, sino que se empieza a develar la imagen de un sujeto político, un artesano de la pedagogía que "produce saber, inventa, crea, innova, genera nuevas propuestas de relación con los contextos, los saberes y los actores educativos y las comunidades" ((Martinez, Leudo, \& Martínez de Rueda, 2005) Las condiciones que hacen posible la configuración de maestros que piensen lo vivo, la vida y su enseñanza y esa relación inmanente entre lo visible y lo decible, la teoría y la práctica, enmarcan a un maestro que a través de espacios como la plenaria, ${ }^{4}$ los ambientes de formación, los ciclos de profundización y los planos ético-políticos toma los insumos que tiene a su alrededor para moldearlos. Es un sujeto que produce, elabora, descifra, genera resistencias, es un intelectual que hace cuestionamientos y asume la biología no desde el lugar del científico o del biólogo, sino que, teniendo en cuenta las realidades educativas, estructura los procesos de enseñanzas y pone en acción un saber que le es propio, un saber complejo, un saber que alimenta la pedagogía. "El maestro, como sujeto de la práctica pedagógica, construye categorías, conceptos, nociones y objetos de saber para redefinir a través de estos

4 Actividad académica realizada por todos los participantes en el desarrollo de un eje curricular particular (profesores, estudiantes, monitores, auxiliares). Con ella se pretende, entre otras cosas, posibilitar el mejoramiento de la interacción en el equipo, la planeación y la evaluación conjunta de las actividades, así como la discusión de problemas pedagógicos que surjan de la confrontación entre la teoría y la praxis. elementos su relación con la educación, con las políticas educativas, con la enseñanza y con la cultura" (Martinez Boom, 2004, p. 23).

\section{Prácticas corporales: la pedagogía como incitación}

En este segundo pliegue (figura 2) el archivo muestra como temáticas fuertes la construcción de saber, cuerpo, experiencias, corporalidad, territorio. Dichas categorías reorganizan las prácticas de enseñanza, le dan un lugar privilegiado a la pedagogía y permiten situar la noción de cuerpo de forma distinta. Pues, en este segundo momento, la pregunta por la formación del cuerpo se redirige desde la perspectiva biomédica para entrar en una lógica que incluye la vivencia y el sentir corporal. En este marco, la pedagogía bordea y produce nuevas formas de sentir, regular y visibilizar el cuerpo teniendo en cuenta la formación integral que se estructura en los espacios del proyecto curricular de la Licenciatura en biología.

La mirada sobre el cuerpo transita y se ubica de forma distinta en cada momento histórico. Inicialmente, en la Licenciatura se encaminó la enseñanza de los futuros profesores en biología a la educación sanitaria, cuidado del cuerpo, ligado a prácticas y métodos de higiene para lograr salud y éxito personal. Pero a partir de sucesos como la expedición pedagógica señalada anteriormente y de saberes como la antropología y la diversidad cultural, se empiezan a instaurar otras formas de entender el cuerpo y la configuración de subjetividades.

Esto refleja un cambio en la idea del gobierno de sí. En un primer momento, la educación se inclina por el cuidado principalmente del cuerpo-espíritu, pero, posteriormente, el cuerpo se ve ligado a la experiencia, al territorio, a la producción de saberes, al sentir corporal. La dimensión humanística y pedagógica viabiliza otros discursos sobre el cuerpo anudados a la salud como subjetivación, a las prácticas alimentarias, a la interacción entre algunos procesos vitales: relaciones, placer, roles sociales e identidad, se produce un cuerpo enmarcado en algunos postulados de la escuela activa en torno a la experiencia.

Aspectos como estos circularon en la Licenciatura a través de referentes como el texto Escenarios de la corporeidad, de Duch y Melich, e Historia de la sexualidad (2002, 2005), de Michel Foucault. Al igual que las publicaciones y trabajos de la línea de investigación trayectos y aconteceres y seminarios como Escuelay sexualidad: otros cuerpos, otras sexualidades (2004); Fisiología de la alimentación y prácticas alimentarias en la escuela (2004) y Naturaleza, cuerpoy cultura, en este último, se destaca el siguiente fragmento: 
La idea es reconocer la corporalidad no solo como un fenómeno netamente biológico, sino también como un fenómeno eminentemente cultural y en tal sentido, el papel determinante que en el proceso de su constitución han jugado tanto el mecanismo evolutivo de la selección natural como las acciones, intervenciones, concepciones y representaciones sociales. (Guzmán, 2004)

Igualmente, se empiezan a elaborar trabajos de grado que tiene como eje fundamental la experiencia y su incidencia en el aprendizaje, en las prácticas corporales y en el pensamiento reflexivo. Uno de ellos es Aprender por la experiencia: Una aproximación al pensamiento de John Dewey (2004), en el cual se establece como conclusiones que "la intención del manejo de experiencias dentro de la escuela es permitir que el conocimiento tenga un carácter trascendental y menos inmediato" (p. 14).

Con lo anterior, la pedagogía entra como fuerza de incitación que al permear la biología, anuda nuevas categorías que permiten situar la noción de cuerpo, experiencia, vivencia corporal, la enseñanza, las prácticas y los contenidos de forma distinta en la Licenciatura.

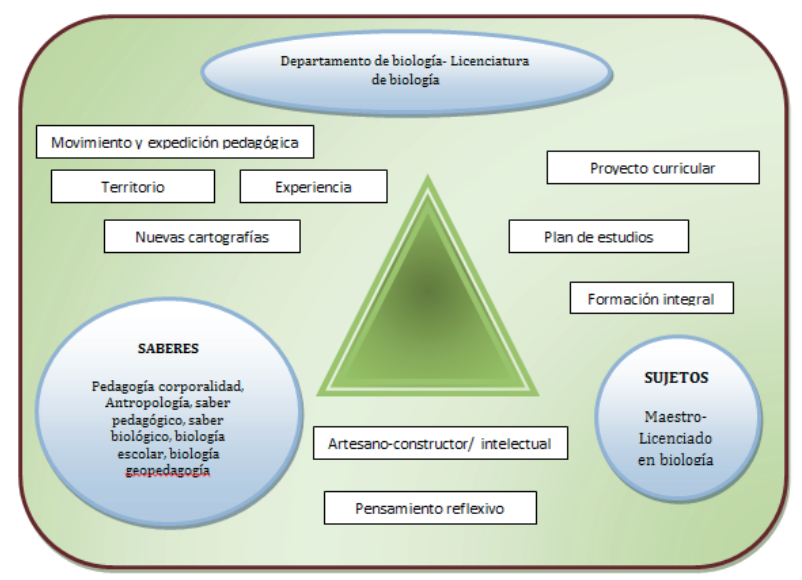

Figura 2. Segundo pliegue, Nuevas cartografías, nuevas pedagogías

\section{Tercer pliegue. Encuentro y desencuentro de saberes}

El tercer momento aparece como un conglomerado de hechos y sucesos difíciles de comprendery descifrar, pero, a raíz de la tematización, emergió un espacio temporal, en el cual esa acumulación empezó a cobrar sentido.

Las relaciones establecidas entre la Licenciatura y la sociedad se hacían más claras, el hoy está construido por una diversidad tan amplia que aparecen líneas en todas las direcciones (una especie de caos). La Licenciatura, a través del diálogo y la reflexión, propende por unos espacios académicos en los que se encuentren, organicen y respeten las distintas realidades; sin imponer y jerarquizar conocimientos.

Aunque son evidentes las tensiones, matices y resistencias que se anudan en cada proceso de la licenciatura, es a través del recorrido realizado en este pliegue donde la pedagogía se ubica como eje articulador que posibilita el encuentro de acciones, la posibilidad de pedagogías propias, las prácticas reflexivas y de afecto5 en el ejercicio docente.

\section{Docente de biología, gestor de cultura.}

La transformación en la manera de concebir lo vivo y los avances significativos de la ciencia, la tecnología y la sociedad han acarreado una serie de exigencias en la formación académica de los futuros docentes en todas las áreas del sector educativo, una de esas áreas es biología o ciencias naturales. Además, con los discursos en torno a la diversidad étnica y cultural 6 se genera la necesidad de asumir un enfoque diferencial que valore y reconozca la diversidad y los saberes ancestrales y que promueva una ética ambiental para evitar la degradación de los ecosistemas.

Estas connotaciones se evidencian en varias de las reflexiones que ha adelantado el Departamento de Biología en lo referente al perfil profesional y las formas de apropiación del conocimiento biológico, no solo en la sede central, sino también en sus tres extensiones y los ceres de La Chorrera, Amazonas; Puerto Asís; Puerto Caicedo, Putumayo, que articulados promueven una formación de docentes a partir del reconocimiento de la interculturalidad, la inclusión y la diversidad.

5 La noción de afecto se toma a partir de la mirada del curso electivo "Educación en el afecto" propuesto por la profesora Paola Roa García, en el cual se visibiliza cómo el afecto no solo responde a la noción de ternura o niñez, sino que el afecto está relacionado con la posibilidad de afectar a sí mismo y al otro, lo que asume el afecto como prácticas de poder y resistencia

6 El principio de protección de la diversidad étnica y cultural es una manifestación directa del pluralismo, que, a su vez se inspira en la tolerancia y el respeto activo, y que abre un espacio de inclusión y participación a grupos tradicionalmente discriminados, como lo son las minorías culturales, raciales, ideológicas, sexuales, religiosas y de cualquier otra índole. http://www.banrepcultural.org/blaavirtual/constitucion/ diversidad-etnica-y-cultural 
Además con el proceso de formación integral se destaca el desarrollo de pensamiento crítico y la capacidad de transformación de las prácticas docentes (praxis educativa) que tenga en cuenta escenarios como la escuela. Retomando a (Bourdieu, \& Passeron 1981) y (Bernstein 1990), la escuela se instaura como un dispositivo de poder que ordena y jerarquiza, es decir, tiene claramente definidos los roles y las reglas de comportamiento. Por lo tanto, es relevante la formación de un docente que reflexione acerca de las formas de conocimientos que circundan en la escuela, que logre identificar los discursos de poder/saber que se instauran. Pues, haciendo alusión a lo propuesto por Michel Foucault, citado en: (Mclaren, 1994) "el poder se le construye socialmente, forma parte de una política cultural y sirve a intereses estructurados dentro de la sociedad". Así, el poder produce formas de conocimiento que subjetivan pero que también pueden subyugar.

Aunque no se puede desconocer que con las reformas educativas y el Estatuto Docente 1278 de 2002 el maestro queda sujeto a dictámenes donde otras disciplinas "expertas" ajenas a la realidad cotidiana del aula y de los procesos de enseñanza-aprendizaje se disputan el campo educativo, es evidente que con los discursos de dignificación académica se busca encarar estos retos y posicionar el conocimiento profesional del profesor de ciencias, desde distintas áreas. Una de ellas es la psicología (cognitiva, educativa y del desarrollo) que le brinda herramientas psicopedagógicas para potenciar el desarrollo del pensamiento.

Igualmente, durante la renovación de acreditación de alta calidad del Proyecto Curricular de la Licenciatura en Biología, ocurrida en el 2010, se fortaleció la formación de educadores con capacidad de comprender y transformar contextos y liderar acciones encaminadas a la valoración social de la profesión docente, desde un compromiso ético que no minimice la mirada y los saberes del otro.

Para el caso de la formación de licenciados en biología, de igual manera, es preciso tener en cuenta que en la formación de los ciudadanos interviene el conocimiento sobre lo vivo en particular y su trascendencia sobre la vida, en relación con diversos saberes, actitudes y valores que configuran, construyen y reconstruyen sentidos y posibilidades en la acción humana. (Departamento de Biología, 2010, p. 9)

En los anteriores elementos se encuentra una fuerte inclinación a formar los docentes de biología desde particularidades socioculturales con una responsabilidad social y política, compromiso con los contextos y los impactos ambientales que promueven nuevas formas de asumir lo vivo y de intervenir la vida. Un docente reflexivo que geste espacios de innovación articulando saberes como la biotecnología y las actitudes científicas de los estudiantes, con estrategias didácticas que favorezcan la enseñanza, de acuerdo al contexto, a su vez, se asume como un sujeto gestor de cultura dentro y fuera del aula de clases, que adopta una actitud respetuosa de la diferencia y la diversidad cultural, étnica y sexual.

\section{Diversidad de experiencias, la pedagogía como eje articulador}

La biología ya no es un saber inofensivo como se pensaba antes, su enseñanza no es sólo para despertar la curiosidad y desarrollar la observación; hoy se ve como un poder que permite al hombre transformar la naturaleza y transformarse así mismo (Host, 1988). Además, involucrada en un periodo de cambios en torno a la enseñanza y el aprendizaje, adquiere puntos singulares que propician diversos saberes, actitudes y valores en relación con las realidades del contexto.

En este tercer pliegue, (figura 3) la pedagogía aparece como eje articulador de una diversidad de experiencias y prácticas en torno a las particularidades socioculturales y la emergencia de nuevos saberes (interculturalidad, biotecnología, saberes ancestrales, educación sexual, sociología de la educación, modelización) lo cual expande tendencias y perspectivas en la Licenciatura en Biología.

Para finalizar este recorrido, nos encontramos con una pedagogía capaz de articular multiplicidad de saberes, en diversos contextos y con la posibilidad de formar a través de la Licenciatura en Biología unos maestros con la capacidad de leer, respetary articular estas diferentes realidades en un "país biodiverso, pluriétnico y multicultural".

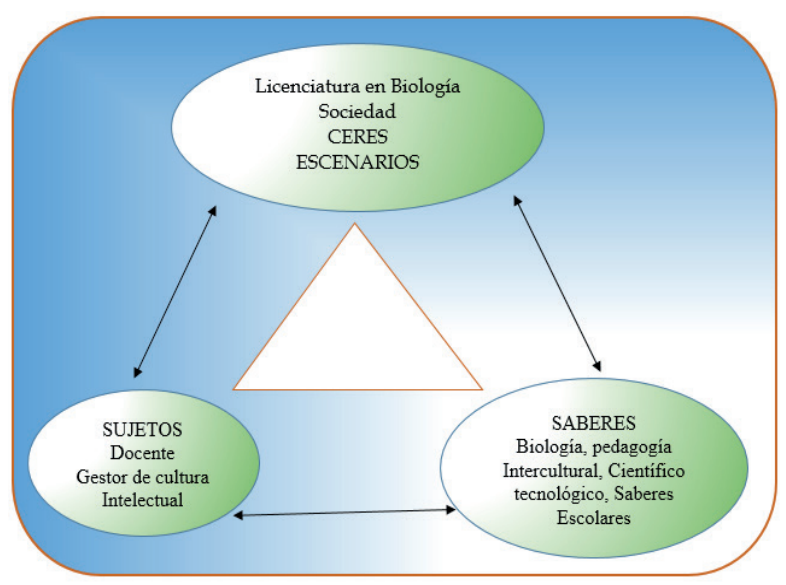

Figura 3: Encuentro y desencuentro de saberes. 


\section{Consideraciones finales}

Aproximarnos a una licenciatura diferente a la nuestra no fue tarea fácil, requirió compromiso, acercamientos a conceptos que desconociamos, vínculos, sospechas y reflexiones a partir de los hallazgos.

Además, teniendo en cuenta varios elementos de la caja de herramientas del Grupo Historia de la Práctica Pedagógica, empezamos a confrontarnos con la idea de no juzgar, de no interpretar, sino de dejarnos asombrar por lo que emergía en los documentos que constituyeron el archivo. Fue tomar distancia para problematizar y a la vez, poco a poco, enredarse, apasionarse y tejer nuevas construcciones alrededor de la relación entre la biología y la pedagogia, que se enmarcaron en la Licenciatura en Biología.

Inicialmente, el primer pliegue muestra una enseñanza de la biología enmarcada en la cuestión de la higiene, educar es higienizar. En este sentido, la pedagogía se ubica como una estrategia para la prevención y promoción de las prácticas higiénicas en aras de un individuo, una familia y una comunidad saludable con miras al progreso socioeconómico del país. Por lo tanto, se necesitaba intervenir los sujetos, especialmente los niños, con un conjunto de prácticas para mejorar los estados de salud y lograr una educación del cuerpo. Esto se hacía mediante una capacitación adecuada de los educadores para la salud que actuaran como vigías de normas higiénicas y hábitos saludables. Además, la educación para la salud fue tomando fuerza en las universidades, en correspondencia con las demandas que hacia la sociedad para evitar más enfermedades infecciosas que hicieran decaer el proyecto de una sociedad fuerte y sana, ligada al bienestar socioeconómico del país.

El segundo momento y pliegue evidenció una relación entre la biología y la pedagogía enmarcada en las elaboraciones que suscitó el movimiento y la expedición pedagógica. En conexión con reflexiones adelantadas por los profesores sobre su práctica y el modelo educativo que había imperado en el plan de estudios, se produjo una revolución pedagógica dentro del Departamento de Biología que permitió situar la pedagogía como una fuerza de incitación que generó trazos, cartografías y develó posibilidades de asumir la enseñanza de la biología de forma diferente, no subordinada a estrategias científicas o reproductora de prácticas conductistas. Con estas connotaciones se dio un viraje entorno a lo disciplinar, pedagógico y didáctico que llevó a posicionar al maestro como artesano de la pedagogía que produce y construye saberes. Además, la pedagogía, al bordear y producir nuevas formas de sentir, regular y visibilizar el cuerpo teniendo en cuenta la formación integral estructurada en los espacios del proyecto curricular de la Licenciatura en Biología, empezó a posicionar la noción de cuerpo desde la experiencia ligado a nociones como territorio, vivencias y diversidad sexual.

Finalmente, el tercer pliegue anclado en la diversidad y encuentro y desencuentro de saberes reveló una relación entre la pedagogía y la biología enmarcada en múltiples tendencias que circulan y permean la enseñanza de los futuros docentes de biología, no solo en la sede central de la Licenciatura, sino también en sus tres extensiones y los ceres, que promueven una formación a partir del reconocimiento de la interculturalidad, la inclusión y la diversidad.

En este caso la pedagogía entra como eje articulador que anuda distintos saberes y posibilita diversidad de experiencias. Pero a la vez genera inquietud y prácticas reflexivas acerca del docente de biología como gestor de cultura, que está imbricado en demandas que recaen a nivel social sobre su profesión y que le exigen un compromiso ético con la biodiversidad. En este panorama, la pedagogía contribuye y suscita nuevos interrogantes acerca de lo vivo y la vida; se preocupa por el sujeto y el lugar que ocupa en la sociedad en una amalgama con la biología.

Con lo anterior, y a raíz de estos pliegues, se enmarca los matices que ha tenido la Licenciatura en Biología configurando enunciados, relaciones de poder y saber instauradas en cambios culturales, económicos, políticos, ambientales, educativos. Estos matices con transformaciones y prácticas particulares denotan una complejidad y unas condiciones de emergencia que le dan una identidad única al programa de formación en biología de la Universidad pedagógica y a los maestros que en él se forman.

Asimismo, sin pretender resolver inquietudes ni establecer fórmulas de acción, se evidencian las discusiones, rutas de análisis y resistencias suscitadas desde el interior del proyecto curricular sobre la enseñanza de la biología, el conocimiento profesional del profesor y las tensiones, reflexiones políticas, éticas y estéticas que permiten situar la formación de maestros y la relación entre lo disciplinar y la pedagogía de forma distinta. 


\section{Referencias}

Bautista, T. A. (1979). Programa de didáctica de ciencia integrada a nivel preescolar, Ficha Tematización 8. Departamento de biología. Bogotá.

Castro, J. A., y Valbuena, É. O. (2007). ¿Qué biología enseñar y cómo hacerlo? Hacia una resignificación de la Biología escolar. Tecné Episteme y Didaxis, No 22, 126-145.

Chona D. G., Castaño, N. C., Cabrera, F. M., Arteta de Molina, J., Valencia, S., \& Bonilla, P. A. (1998). Lo que nos dice la historia de la enseñanza de la biología en Colombia. Una aproximación. En: Tecne, Epistemey Didaxis, pág., 5-10.

Chona, D. G. (2014). Grabado por G. C. Hernández, y A. S. Cano. Entrevista 01. Universidad Pedagógica Nacional, Bogotá.

Departamento de Biología y Química (1961). Programa correspondiente a Biología y Química, Ficha Tematización 2. Universidad Pedagógica Femenina. Bogotá.

Departamento de Biología (1973). Programa de educación para la salud, $7 .^{\circ}$ y $8 .^{\circ}$ semestre de Biología y Edufísica, Ficha Tematización 3. Bogotá.

Departamento de Biología (1985). Plan de estudios (semáforo), Ficha Tematización 2. Universidad Pedagógica Nacional. Bogotá.

Departamento de Biología (1999). Proyecto curricular, Licenciatura en Biología. Universidad Pedagógica Nacional. Bogotá.

Departamento de Biología (2010). Informe renovación de acreditación de alta calidad del Proyecto Curricular de la Licenciatura en Biología, Ficha Tematización 77. Universidad Pedagógica Nacional. Bogotá.

Guido, A., y Arias, H. (1985). Programa de Biología general para psicopedagogía, Ficha Tematización 11. Departamento de Biología. Bogotá.

Guzmán, H. (2004) Programa curso electivo: Naturaleza, cuerpo y cultura. Ficha Tematización 47. Departamento de Biología.

Martínez, S., Leudo, M., \& Martínez de Rueda, S. (24 de Octubre de 2005). Propuesta curso electivo expedición por las prácticas de la enseñanza. Bogotá: Departamento de Biología, Universidad pedagógica Nacional. Ficha tematización No 55.
Olivares, A., Luna, J., \& Delgado, A. J. (s. f.). Programa de post-grado en educación para la salud. Área de salud mental Universidad Pedagógica Nacional. Bogotá.

Pizarro de Gómez, M. (1978). Programa sobre cuidados básicos y primeros auxilios con el preescolar, Ficha Tematización 5. Departamento de Biología, Facultad de Ciencia y Tecnología. Bogotá.

Roa García, P. A. (2010). Curso electivo educación en el afecto, Centro de Documentación Licenciatura en Biología, Ficha 74. Universidad Pedagógica Nacional. Bogotá.

Rodríguez de Lara, F. (1978). Programa de educación para la salud, Ficha Tematización 6. Bogotá.

Rodríguez de Lara, F., y Herrera, D. (1994). Propuesta de un programa de posgrado en educación para la salud, Ficha Tematización 25. Departamento de Biología. Bogotá.

Universidad Pedagógica Nacional (2010). Fundamentos conceptuales. Documento de acreditación .Licenciatura en biología Ficha 36. Bogotá.

Universidad Pedagógica Nacional (2014)., Licenciatura en Biología, Plan Curricular. Obtenido de http://cienciaytecnologia.pedagogica.edu.co/vercontenido. php?idp=373\&idh=374\&idn=10007

Valbuena, E., Sierra, C., y Gutiérrez, A. (2007). Una década de investigación en la Facultad de Ciencia y Tecnologia de la UPN. TEA, 146-189.

Werner Cantor, E. (2006) Componente diversidad cultural, Ficha Tematización 56. Departamento de Biología. Bogotá.

Fuentes secundarias

Álvarez A. (2003). La pedagogía y las ciencias: historia de una relación. En O. Zuluaga, A. Echeverri, A. Martínez, H. Quiceno, J. Saenz \& A. Álvarez, Pedagogía y epistemología. Bogotá: Cooperativa Editorial Magisterio.

Berstein, B. (1990). Poder, Educación y conciencia, Barcelona: editorial el Roure

Bourdieu, P \& Passeron, J. (1970/1981) La reproducción elementos para una teoría del sistema de enseñanza. Barcelona: Laia

Dewey, J. (1938/1958). Experiencia y educación. Buenos Aires: Editorial Losada. 
Foucault, M. (1966). La arqueología del saber. México: Siglo xxı editores.

Foucault, M. (1974). La crisis de la medicina o la crisis de la antimedicina. Curso de Medicina Social Universidad Estatal de Rio de Janeiro, Brasil.

Gutiérrez, D. P. (1963). La familia en Colombia. Bogotá: Iqueima.

Host, V., (1988). La historia de la teoría celular. En, Conceptos de Biología Nº 2 Girodan et.al. Barcelona,. Labor.

Martínez Boom, A. (2004). Hacia un atlas de la pedagogía en Colombia. Revista Alternativas, 35-36, 171-184

Martínez Boom, A., Noguera, C., y Castro, J. O. (1994). Currículo y modernización. Cuatro décadas de educación en Colombia. Bogotá: Servigraphic Ltda.

Mclaren, P. (1994) Pedagogía critica, resistencia cultural y la producción del deseo. Buenos Aires. Aique.
Mendoza, J. S. (1965). Puericultura e higiene escolar. México: Trillas, s.a.

Noguera, E. (2003). Medicina y política discurso médico y prácticas higiénicas durante la primera mitad del siglo xx en Colombia. Medellín: Fondo Editorial Universidad EAFIT.

Pedraza Gómez, Z. (2004). Y el verbo se hizo carne. Pensamiento social y biopolítica en Colombia. En Castro S. (ed), Pensar el siglo xx. Cultura, biopolítica y modernidad en Colombia (p. 324). Pittsburgh, University de Pittsburgh

Roa García, P. A. (2011). Historia de la biología en la escuela colombiana: Una mirada genealógica entre 1900 y 1930. En Bio-grafía, pág., 544-559.

Zuluaga, O. L. (1999). Pedagogia e historia: La historicidade de la pedagogia, la enseñanza um objeto de saber. Santafé de Bogotá: Anthropos. Universidad de Antioquia, 